\title{
Evaluación diagnóstica y manejo terapéutico farmacológico en pacientes con fibrilación auricular
}

\author{
Orlando Robert Sequeira ${ }^{1}$, Judith María Torales ${ }^{1,2}$, Laura Beatriz García ${ }^{1,2}$, *Osmar Antonio Centurión ${ }^{1,2}$ \\ ${ }^{1}$ Universidad Nacional de Asunción. Hospital de Clínicas, División de Medicina Cardiovascular. San Lorenzo. \\ Paraguay \\ ${ }^{2}$ Sanatorio Metropolitano. Departamento de Investigación en Ciencias de la Salud. Fernando de la Mora. Paraguay
}

Cómo referenciar este artículo/ How to reference this article:
Sequeira OR, Torales JM, García LB, Centurión OA. "Evaluación diagnóstica y manejo terapéutico farmacológico en pacientes con fibrilación auricular". Mem. Inst. Investig. Cienc. Salud. 2019; 17(2): 86-101

\section{R E S U M E N}

La fibrilación auricular (FA) es la arritmia sostenida más frecuente que puede presentarse en una amplia variedad de condiciones clínicas. A pesar de los progresos en el tratamiento de los pacientes con FA, esta arritmia sigue siendo una de las más importantes causas de accidente cerebrovascular, insuficiencia cardiaca, muerte súbita y morbilidad cardiovascular en todo el mundo. El objetivo del tratamiento con fármacos antiarrítmicos es mejorar los síntomas relacionados con la FA, y buscar un equilibrio entre la carga sintomática, la posibilidad de reacciones farmacológicas adversas y las preferencias del paciente. El control de la frecuencia cardiaca a corto y largo plazo se puede lograr con bloqueadores beta, digoxina, bloqueadores de los canales del calcio o tratamiento combinado. Si bien otros fármacos antiarrítmicos de clase III también tienen un efecto limitador de la frecuencia, solo deben emplearse para el control del ritmo cardiaco. Restaurar y mantener el ritmo sinusal también es una parte integral del tratamiento de la FA. Los fármacos antiarrítmicos de clase III duplican la tasa de pacientes en ritmo sinusal. El tratamiento para el control del ritmo está indicado para mejorar los síntomas de los pacientes con FA que siguen sintomáticos a pesar del tratamiento adecuado para el control de la frecuencia cardiaca. La combinación de antiarrítmicos que prolongan el intervalo QT no se debe emplear para el control del ritmo cardiaco en la FA.

Palabras clave: Fibrilación Auricular, tratamiento farmacológico, agentes anti-arrítmicos.

\section{Diagnostic evaluation and pharmacological therapeutic management in patients with atrial fibrillation}

\section{A B S T R A C T}

Atrial fibrillation (AF) is the most frequent sustained arrhythmia that can occur in a wide variety of clinical conditions. Despite progress in the treatment of patients with $A F$, this arrhythmia remains one of the most important causes of stroke, heart failure, sudden death and cardiovascular morbidity worldwide. The aim of treatment with antiarrhythmic drugs is to improve the symptoms related to $\mathrm{AF}$, and to look for a balance between the symptomatic load, the possibility of adverse pharmacological reactions and the patient preferences. The short and long term heart rate control can be achieved with beta blockers, digoxin, calcium channel blockers or combination therapy. Although other class III antiarrhythmic drugs also have a frequency-limiting effect, they should only be used for the control of heart rhythm. Restoring and maintaining sinus rhythm is also an integral part of the treatment of AF. Class III antiarrhythmic drugs double the rate of patients who maintain their sinus rhythm. Treatment for rhythm control is indicated to improve the symptoms of patients with AF who 
remain symptomatic despite adequate treatment for heart rate control. The combination of antiarrhythmics that prolong the QT interval should not be used to control heart rhythm in AF.

Keywords: Atrial Fibrillation, Pharmacotherapy, Anti-arrhythmic agents.

\section{INTRODUCCIÓN}

La fibrilación auricular (FA) es la arritmia sostenida más común que se encuentra en el campo de la medicina interna, tiene una prevalencia de aproximadamente el $1 \%$ y un riesgo de por vida de aproximadamente el $25 \%$ después de los 40 años ${ }^{(1-3)}$. La fibrilación auricular comparte fuertes asociaciones epidemiológicas con otras enfermedades cardiovasculares como la insuficiencia cardíaca y la enfermedad arterial coronaria ${ }^{(1-5)}$. Muchos aspectos fundamentales de la FA han sido poco conocidos hasta hace muy poco, y hay varias características en los mecanismos de FA que dificultan su gestión adecuada. La FA puede presentarse en una amplia variedad de condiciones clínicas. La estrategia de tratamiento óptima para un paciente individual con FA depende de la afección subyacente del paciente ${ }^{(6-}$ 8).

La fibrilación auricular (FA) es la arritmia cardíaca más común que se caracteriza electrocardiográficamente por oscilaciones basales de baja amplitud (fibrilatorias $u$ ondas $f$ ) y por un ritmo ventricular irregularmente irregular. Las ondas $f$ tienen una frecuencia de 300 a 600 latidos/min y varían en cuanto a amplitud, forma y duración. En la derivación V1, las ondas $\mathrm{f}$ se muestran uniformes y pueden imitar a las ondas de aleteo auricular. El rasgo diferencial respecto del aleteo auricular es la ausencia de actividad auricular uniforme y regular en las otras derivaciones del electrocardiograma. En algunos pacientes, las ondas $f$ son muy pequeñas e imperceptibles en el electrocardiograma. En estos pacientes, el diagnóstico de $F A$ se basa en el ritmo ventricular irregularmente irregular. La frecuencia ventricular durante la FA, en ausencia de agentes dromo-trópico negativos es generalmente de 100 a 160 latidos/min.

Las estrategias de tratamiento de la FA con el control de ritmo o el control de la frecuencia se asocian con tasas similares de mortalidad y morbilidad grave. Sin embargo, se preferiría seguir la estrategia de control del ritmo por la mejoría de los síntomas, en los pacientes más jóvenes y para mejorar la remodelación estructural y eléctrica que puede ocurrir con la FA persistente de larga duración ${ }^{(9-11)}$. La decisión de adoptar un control del ritmo o una estrategia de control de frecuencia a menudo viene dictada por la presencia de síntomas asociados con la fibrilación auricular y/o desarrollo de disfunción sistólica del ventrículo izquierdo como consecuencia de la frecuencia alta de la $\operatorname{arritmia}^{(12-14)}$.

En pacientes con síndrome de Wolf-Parkinson-White, la frecuencia ventricular durante la FA puede superar los 250 latidos/min, debido a la conducción a través de la vía accesoria lo cual puede desarrollar una fibrilación ventricular y muerte súbita ${ }^{(15-19)}$. Cuando la frecuencia ventricular durante la FA es muy rápida, más de 170 latidos/min, el grado de irregularidad tiende a disminuir y el ritmo puede parecer regular ${ }^{(20-25)}$. La FA puede tener consecuencias adversas relacionadas con una reducción en el gasto cardíaco y en la formación de trombos auriculares y atriales. Además, los pacientes afectados pueden estar en mayor riesgo de mortalidad ${ }^{(26-31)}$. La prevalencia de la fibrilación auricular (FA) aumenta con la edad y los ancianos son el subconjunto de la población con el crecimiento más rápido. A pesar de los progresos en el tratamiento de los pacientes con fibrilación auricular (FA), esta arritmia sigue siendo una de las más importantes causas de accidente cerebrovascular, insuficiencia cardiaca, muerte súbita y morbilidad cardiovascular en todo el mundo(7). Por lo tanto, es nuestra intención realizar un análisis de la evaluación diagnóstica y el manejo terapéutico farmacológico actualizado en pacientes con fibrilación auricular.

\section{Evaluación diagnóstica de la FA}

Cuando un paciente refiere palpitaciones y uno encuentra ciertos datos semiológicos que apuntan hacia una fibrilación auricular, debemos corroborar nuestro diagnóstico semiológico con un electrocardiograma (ECG) convencional. Los datos semiológicos que nos obligan a pensar en una fibrilación auricular son: taquicardia, irregularidad, y déficit de pulso.

\section{Electrocardiograma}

EI ECG demuestra una taquicardia irregular generalmente a complejos QRS angostos. EI ECG se usa para verificar la presencia de FA y es necesario para hacer el diagnóstico. La fibrilación auricular tiene las características electrocardiográficas que se describen a continuación. Los intervalos RR no siguen un patrón repetitivo. Han sido etiquetados como 
"irregularmente irregular". Aunque se puede observar actividad eléctrica sugestiva de ondas $\mathrm{P}$ en algunas derivaciones, hay ondas $\mathrm{P}$ distintas ${ }^{(32-34)}$. Por lo tanto, incluso cuando se puede definir una duración del ciclo auricular (el intervalo entre dos activaciones auriculares o el intervalo PP), no es regular y a menudo menos de 200 milisegundos (lo que se traduce en una frecuencia auricular superior a 300 latidos por minuto).

Hay una serie de riesgos potenciales en el diagnóstico electrocardiográfico de la FA. Los errores en el diagnóstico de FA son especialmente comunes con la interpretación computarizada del ECG y en pacientes que se someten a un ritmo continuo o intermitente. Por lo tanto, es importante que la interpretación automatizada de ECG proporcionada por la máquina sea confirmada por un lector experto. Un ECG basal, preferiblemente en ritmo sinusal, también debe evaluarse para obtener la siguiente información que se detalla en la Tabla 1.

Tabla 1: Evaluación electrocardiográfica en ritmo sinusal en pacientes con FA

1. Marcadores de enfermedad cardíaca no eléctrica como hipertrofia ventricular izquierda por posible hipertensión arterial.

2. Marcadores de enfermedad cardíaca no eléctrica como ondas Q por posible enfermedad coronaria.

3. Marcadores de enfermedad cardíaca eléctrica como el síndrome de preexcitación ventricular o síndrome de Wolff-Parkinson-White.

4. Marcadores de enfermedad cardíaca eléctrica como enfermedad de conducción infranodal por bloqueo de rama derecha o izquierda.

5. Prolongación del intervalo QT para identificar el riesgo potencial de terapia antiarrítmica como efecto proarrítmico.

6. Evidencia de bradicardia severa o pausas.

7. Disfunción del nódulo sinusal.

\section{Ecocardiografía Doppler}

El ecocardiograma tras-torácico (TTE) se realiza para evaluar el tamaño de las aurículas derecha e izquierda y el tamaño y la función de los ventrículos derecho e izquierdo; para detectar posibles enfermedades valvulares del corazón, hipertrofia ventricular izquierda y enfermedad pericárdica; y para evaluar la presión pico del ventrículo derecho. EI ETT también puede identificar el trombo auricular izquierdo, aunque la sensibilidad es baja. La ecocardiografía tras-esofágica es mucho más sensible para identificar trombos en la aurícula izquierda o la orejuela auricular izquierda (Figura 1) y se puede usar para determinar la necesidad de anticoagulación antes de cualquier intento de cardioversión farmacológica o eléctrica ${ }^{(35-37)}$.
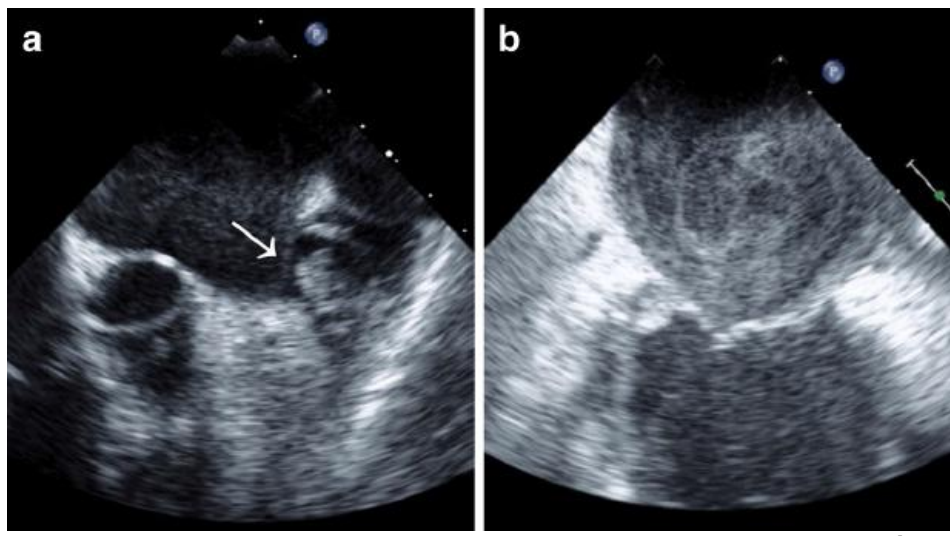

Figura 1: Ejemplos representativos de A) un trombo en la orejuela de la aurícula izquierda, y de B) contraste espontáneo ecocardiográfico en la aurícula izquierda. Reimpreso con permiso de Pant R, Patel M, Garcia-Sayan E, Wassouf M, D'Silva O, Kehoe RF, Doukky R. Impact of B-type natriuretic peptide level on the risk of left atrial appendage thrombus in patients with nonvalvular atrial fibrillation: a prospective study. Cardiovascular Ultrasound 2016;14:4 DOI 10.1186/s12947-0160047-6.

\section{Pruebas cardiacas adicionales}

La prueba de esfuerzo es un método auxiliar de diagnóstico muy beneficioso para pacientes con signos o síntomas de la enfermedad isquémica del corazón. También es útil 
para ayudar a guiar la farmacoterapia para la FA, ya que algunos medicamentos antiarrítmicos están contraindicados en pacientes con enfermedad coronaria. Además, las pruebas de estrés pueden ser útiles para evaluar cuan eficaz es la dosis anti-arrítmica para el control de la frecuencia cardíaca en la FA durante el ejercicio. El control insuficiente de la frecuencia cardíaca en la FA es un factor importante para la intolerancia al ejercicio en la $F A^{(12)}$.

La monitorización cardiaca ambulatoria con registradores de eventos, monitores adhesivos de eventos de tiempo extendido o monitores implantables de bucle (loop recorder) se puede utilizar para identificar la arritmia si es intermitente y no se registra y documenta en la electrocardiografía de rutina. La monitorización ECG ambulatoria también se puede utilizar para correlacionar los síntomas con la arritmia junto con la evaluación de la carga de FA. El monitoreo ECG ambulatorio Holter de 24 horas a 48 horas ayuda principalmente en la evaluación de las tasas de respuesta ventricular general en individuos en los que se ha elegido una estrategia de control de frecuencia y existe preocupación por el control inadecuado de la frecuencia cardíaca o la bradicardia( ${ }^{(38-40)}$.

\section{Pruebas de laboratorio de referencia}

Existe una clara asociación entre el hipertiroidismo y la FA. El hipertiroidismo clínico o subclínico está presente en menos del 5 por ciento de los pacientes con FA. Se debe obtener los niveles séricos de la hormona estimulante de la tiroides (TSH) y niveles de T4 libre en todos los pacientes con un primer episodio de FA o en aquellos que desarrollan un aumento en la frecuencia de FA. Otras pruebas iniciales importantes incluyen un hemograma completo, una creatinina sérica, un análisis de proteinuria y una prueba para la diabetes mellitus.

\section{Manejo terapéutico farmacológico}

En la práctica clínica rutinaria, los médicos se pueden encontrar con dos tipos de pacientes con fibrilación auricular: aquellos con FA recientemente diagnosticada y aquellos que han sido diagnosticados y manejados previamente. El cuidado del primero incluye decisiones con respecto a la necesidad de anticoagulación y la elección entre estrategias de control de ritmo o frecuencia. Para los pacientes con diagnóstico establecido, es necesaria una evaluación periódica para evaluar y controlar la eficacia del tratamiento implementado.

Se debe determinar el estado electrolítico y la funcionalidad renal de cada paciente antes de iniciar el tratamiento farmacológico y periódicamente a partir de ese momento. La hipopotasemia o la hipomagnesemia pueden promover el desarrollo de arritmias cardiacas inducidas por el fármaco, mientras que una función renal alterada puede dar como resultado niveles de fármaco en suero superiores a los previstos. El inicio de la terapia con digoxina se ha dividido en una digitalización rápida o lenta seguida de la dosis de mantenimiento con digoxina, y los regímenes propuestos varían considerablemente. Ciertos principios deben considerarse como una guía general para el uso de agentes anti-arrítmicos por los efectos inotrópicos, dromotrópicos o electrofisiológicos, que deben modificarse de acuerdo con las circunstancias clínicas ${ }^{(41)}$. Los pacientes con buena fracción de eyección que reciben fármacos para el control de la frecuencia ventricular en la FA generalmente requerirán una carga más rápida que los pacientes con insuficiencia cardíaca. Algunos pacientes con fibrilación auricular pueden necesitar atención en un centro de atención de agudos o en una unidad de internación de un hospital. Las indicaciones comunes para la atención en una o ambas de estas instalaciones se incluyen en la Tabla 2.

Tabla 2: Indicaciones comunes de internación en pacientes con FA

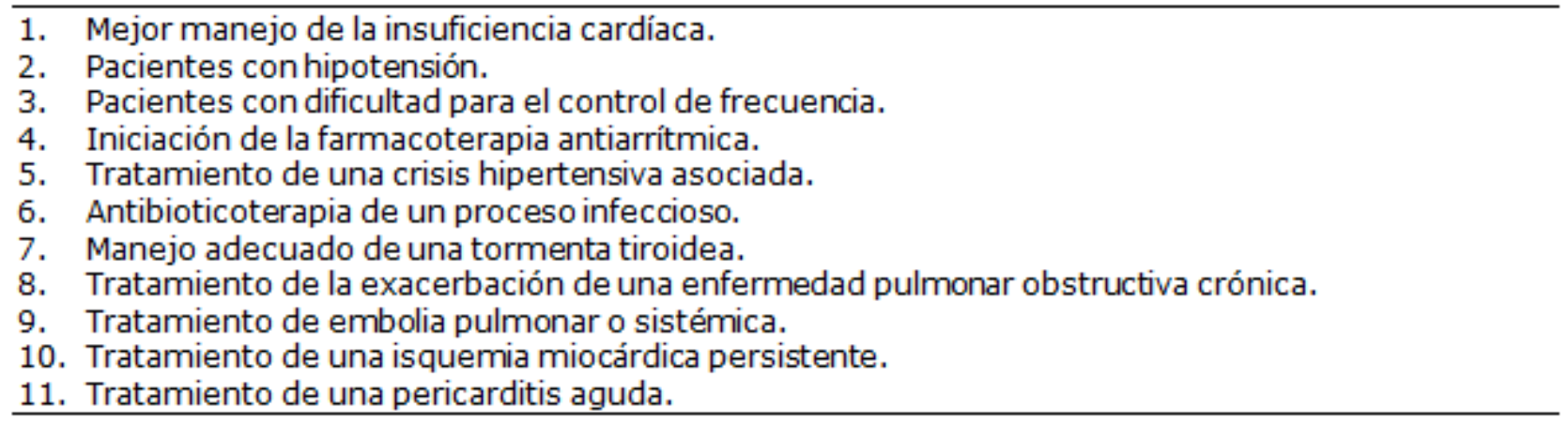




\section{Nueva aparición de FA}

La mayoría de los pacientes con FA de reciente comienzo, es decir, detectado o diagnosticado por primera vez, presentan síntomas relacionados con la arritmia. Excepto por la embolización, los síntomas asociados con la aparición nueva de FA se deben principalmente a una respuesta ventricular rápida. En estos pacientes sintomáticos por una FA de rápida conducción auriculoventricular se ha encontrado una clara asociación con niveles séricos elevados del péptido natriuretico tipo-B (BNP), especialmente en aquellos pacientes con disminución de la funcionalidad ventricular. Pant $R$ et al $^{(37)}$ observaron que se existe una relación directamente proporcional entre los niveles séricos de BNP y la prevalencia de trombos en la aurícula izquierda de pacientes con FA no valvular (Figura 2 y Figura 3). Muchos pacientes tienen una disminución significativa de los niveles séricos de BNP y una mejoría importante en su sensación de bienestar cuando la frecuencia ventricular se reduce con el tratamiento instituido ${ }^{(37)}$. Hay dos cuestiones amplias de manejo que deben abordarse temprano en pacientes con FA de nuevo inicio: la prevención de embolización sistémica y la elección entre una estrategia de control de ritmo o control de frecuencia, que pueden mejorar los síntomas ${ }^{(42)}$.

Se debe evaluar a todos los pacientes con fibrilación auricular para detectar la necesidad de una terapia antitrombótica para prevenir la embolización sistémica incluso en el primer episodio de FA. Esto se logra mediante el uso de la puntuación del Score de $\mathrm{CHA}_{2} \mathrm{DS}_{2}$ VASC. Los pacientes que requieren terapia antitrombótica incluyen aquellos en los que se considera la cardioversión al ritmo sinusal y aquellos que cumplen con los criterios de anticoagulación a largo plazo. Todos los pacientes cuyo riesgo de embolización excede el riesgo de hemorragia son candidatos para la terapia antitrombótica a largo plazo ${ }^{(43)}$.

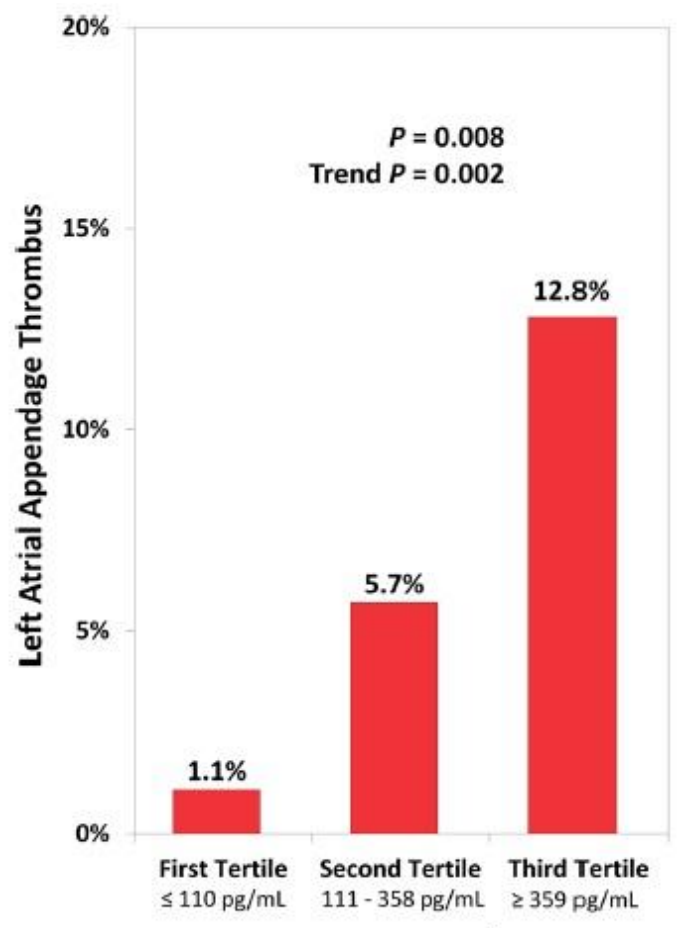

BNP Level

Figura 2: Prevalencia de trombo en la orejuela de la aurícula izquierda de acuerdo al nivel de BNP. Reimpreso con permiso de Pant R, Patel M, Garcia-Sayan E, Wassouf M, D'Silva O, Kehoe RF, Doukky R. Impact of B-type natriuretic peptide level on the risk of left atrial appendage thrombus in patients with nonvalvular atrial fibrillation: a prospective study. Cardiovascular Ultrasound 2016;14:4 DOI 10.1186/s12947-016-0047-6. 


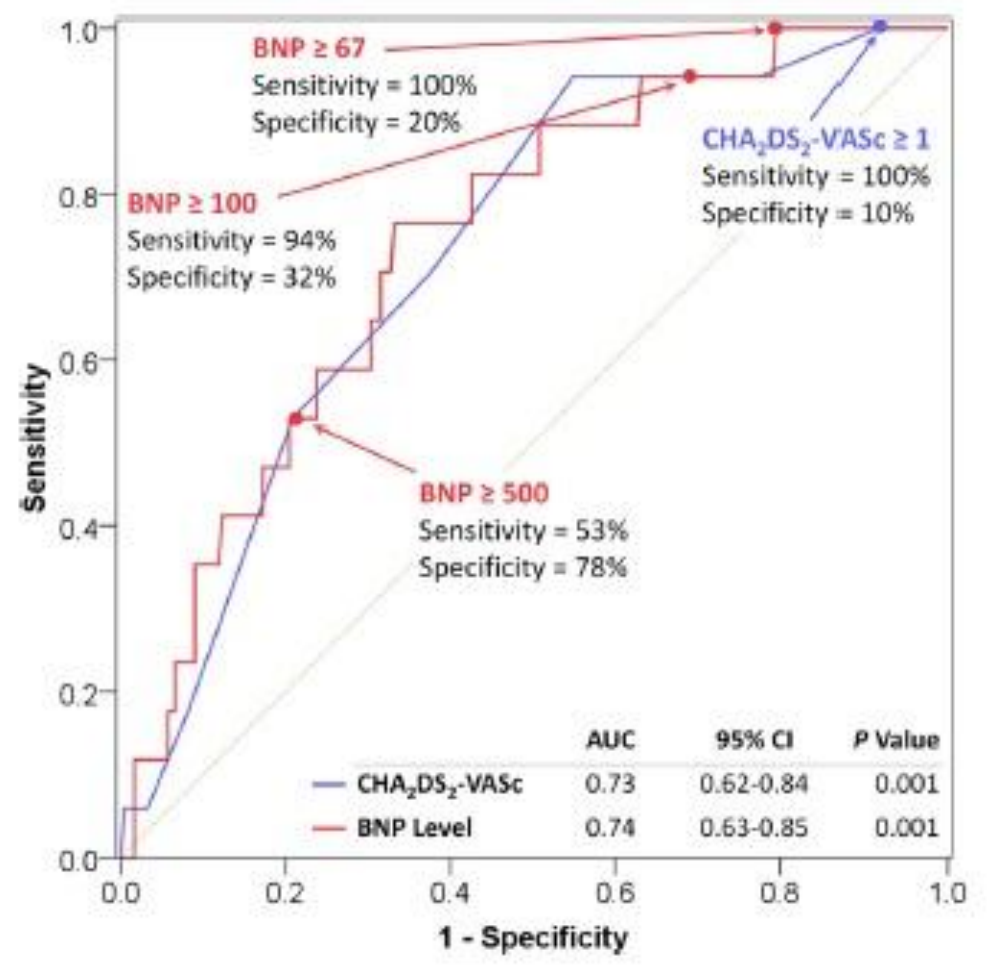

Figura 3: En la figura se pueden observar las curvas diagnósticas características de la presencia de trombo en la orejuela de la aurícula izquierda con su correspondiente sensibilidad y especificidad. Reimpreso con permiso de Pant R, Patel M, Garcia-Sayan E, Wassouf M, D'Silva O, Kehoe RF, Doukky R. Impact of B-type natriuretic peptide level on the risk of left atrial appendage thrombus in patients with nonvalvular atrial fibrillation: a prospective study. Cardiovascular Ultrasound 2016;14:4 DOI 10.1186/s12947-016-0047-6. LAA: apéndice auricular izquierdo. AUC: Área debajo de la curva.

CI: Intervalos de confianza.

\section{Control de ritmo versus frecuencia}

Se ha observado que la mayoría de los pacientes que presentan FA requerirán una disminución de la frecuencia ventricular para mejorar los síntomas. Una vez que se logra el control de la frecuencia ventricular, se debe tomar una decisión con respecto al manejo a largo plazo de la $\mathrm{FA}^{(44-47)}$. Una estrategia de control del ritmo utiliza una terapia con medicamentos antiarrítmicos, ablación percutánea con catéter y/o cirugía cardiaca antiarrítmica. Para lograr restaurar el ritmo sinusal, la cardioversión eléctrica puede ser necesaria. Los medicamentos antiarrítmicos generalmente se inician antes de la cardioversión y continúan manteniendo el ritmo sinusal. Una estrategia de control de la frecuencia generalmente usa medicamentos que disminuyen la conducción a través del nodo auriculoventricular, como los beta-bloqueadores, los bloqueadores de los canales de calcio que no son dihidropiridínicos o la digoxina ${ }^{(44)}$.

Los datos sugieren que las estrategias de control de ritmo y frecuencia se asocian con tasas similares de mortalidad y morbilidad grave. Sin embargo, hay varias razones por las cuales se preferiría seguir una estrategia de control del ritmo. Entre ellas, la mejora de los síntomas, pacientes más jóvenes y la remodelación estructural y eléctrica irreversible que puede ocurrir con la FA persistente de larga duración ${ }^{(45)}$. La decisión de adoptar un control del ritmo o una estrategia de control de frecuencia a menudo viene dictada por la presencia de síntomas asociados con la FA y/o desarrollo de disfunción sistólica del ventrículo izquierdo como consecuencia de la frecuencia alta de la arritmia. Algunos pacientes con FA de nueva aparición que informan estar asintomáticos pueden presentar algunos síntomas sutiles, como fatiga y palpitaciones leves. Estos síntomas más sutiles a veces solo se detectan después de la restauración del ritmo sinusal ${ }^{(45-47)}$.

\section{FA paroxística, persistente, de larga duración, o permanente}

Los pacientes con FA paroxística, persistente, de larga duración o permanente necesitarán atención periódica así como una evaluación urgente ocasional durante la historia natural de su enfermedad. Se sugiere un seguimiento de rutina cada 12 meses en pacientes estables y antes si hay cambios en los síntomas. Los pacientes que reciben terapia antiarrítmica de alto riesgo, como dofetilida o sotalol, a menudo se ven 
cada seis meses. Los pacientes deben realizarse un control de rutina para controlar ciertos parámetros especificados en la Tabla 3.

Tabla 3: Control rutinario de ciertos parámetros clínicos

1. Eficacia y seguridad de la terapia antitrombótica (International Normalized Ratio para pacientes con warfarina).

2. Clearance de creatinina para pacientes en terapia antiarrítmica y con otros anticoagulantes más nuevos.

3. Estado funcional, incluido el cambio en los síntomas (historial médico).

4. Eficacia y seguridad del tratamiento farmacológico antiarrítmico.

5. Electrocardiograma convencional.

6. Evaluación de la función renal y hepática, y quizás otras pruebas.

7. Eficacia del control de la frecuencia.

8. Holter ECG ambulatorio.

La atención de urgencia es necesaria en pacientes que presentan síntomas o signos de FA sintomática. Además de evaluar la eficacia del control de la frecuencia o el control del ritmo, es posible que el médico necesite evaluar y controlar los cambios en los síntomas y signos de la enfermedad arterial coronaria o la insuficiencia $\operatorname{cardíaca}^{(46)}$.

\section{Tratamiento farmacológico para el control de la frecuencia}

El control de la frecuencia es una parte integral del tratamiento de los pacientes con FA $y$, normalmente, es suficiente para mejorar los síntomas relacionados con ella. Comparado con la prevención de los ACV y el control del ritmo cardiaco, hay poca evidencia para establecer el tipo y la intensidad del control de la frecuencia cardiaca más adecuados, y la mayoría de los datos proceden de estudios observacionales o estudios randomizados a corto plazo(44-47).

El control de la frecuencia cardiaca a corto y largo plazo se puede lograr con tratamiento farmacológico, que incluye bloqueadores beta, digoxina, bloqueadores de los canales del calcio (diltiazem y verapamilo) o tratamiento combinado. Si bien otros fármacos antiarrítmicos también tienen un efecto limitador de la frecuencia (amiodarona, dronedarona, sotalol $y$, en cierta medida, la propafenona), solo deben emplearse en pacientes que necesitan tratamiento para el control del ritmo cardiaco ${ }^{(48-68)}$.

\section{Bloqueadores beta}

Los bloqueadores de los receptores beta-adrenérgicos en monoterapia son los fármacos de primera línea para el control de la frecuencia cardiaca, ya que, según los datos disponibles, son más efectivos que la digoxina para el control agudo de la frecuencia cardiaca. Cabe destacar que el beneficio pronóstico de los beta-bloqueadores para los pacientes con IC-FEr en ritmo sinusal se pierde en los pacientes con FA ${ }^{(7)}$. En un metaanálisis de datos individuales, los beta-bloqueadores comparados con placebo no redujeron la mortalidad por todas las causas de los pacientes con FA basal, mientras que se observó un claro beneficio en pacientes en ritmo sinusal.

\section{Bloqueadores de los canales del calcio no dihidropiridínicos}

El verapamilo y el diltiazem proporcionan un razonable control de la frecuencia cardiaca de los pacientes con FA. Se debe evitar en pacientes con IC-FEr debido a su efecto inotrópico negativo. Pueden mejorar los síntomas relacionados con la arritmia comparada con los beta-bloqueadores, que reducen la capacidad de ejercicio y aumentan el péptido natriurético cerebral según los datos de un pequeño estudio de pacientes de bajo riesgo con IC-FEc ${ }^{(7)}$.

\section{Digital}

Los glucósidos cardiacos, como la digoxina y la digitoxina, se han empleado durante más de 2 siglos, aunque la prescripción de estos fármacos ha ido disminuyendo paulatinamente en los últimos 15 años. En el estudio DIG, la digoxina comparada con placebo no tuvo efecto alguno en la mortalidad de los pacientes con IC-FEr en ritmo sinusal, pero redujo el número de ingresos hospitalarios. No se han realizado estudios comparativos directos con digoxina en pacientes con FA. Según los datos de estudios observacionales, el uso de digoxina se asocia a un exceso de mortalidad en los pacientes con FA, pero esta asociación probablemente se deba a sesgos en la selección de los pacientes y la prescripción del fármaco, más que a un efecto nocivo, especialmente debido a que la digoxina se suele administrar a pacientes más enfermos ${ }^{(8)}$. 
En un estudio con grupos cruzados que incluyo a 47 pacientes con IC-FEr y FA, no hubo diferencias en la frecuencia cardiaca, la presión arterial, la distancia recorrida y la fracción de eyección del ventrículo izquierdo (FEVI) entre el carvedilol y la digoxina, pero el bloqueador beta produjo concentraciones más altas de péptido natriurético cerebral; la combinación de carvedilol y digoxina mejoro la FEVI y la retirada de la digoxina la redujo ${ }^{(9)}$. Las comparaciones con otros tratamientos para el control de la frecuencia cardiaca derivan de estudios pequeños y de corta duración en los que no se identificaron diferencias, o estos solos eran marginales, con respecto a la capacidad de ejercicio, la calidad de vida o la FEVI, comparados con la digoxina. La digoxina en dosis bajas ( $\leq 0,25 \mathrm{mg} /$ día), que se corresponden con concentraciones séricas de digoxina de $0,5-0,9 \mathrm{ng} / \mathrm{ml}$, se podría asociar a un mejor pronóstico(7).

La digoxina es un fármaco bien establecido en la medicina cardiovascular, que se emplea ampliamente en los pacientes con $\mathrm{FA}^{(2)}$. Las directrices americanas para el manejo de la FA recomiendan el uso de digoxina, con grado de recomendación I (nivel de evidencia C), para alcanzar el control de la frecuencia cardiaca (FC) en pacientes con insuficiencia cardíaca y FEVI reducida. Con grado de recomendación IIa (nivel de evidencia B), su uso en combinación con otros fármacos, como betabloqueantes o calcio-antagonistas no dihidropiridínicos, para pacientes con insuficiencia cardíaca y FE preservada para el control de la FC en reposo y durante el ejercicio. Las directrices europeas para el manejo de la FA recomiendan el uso de digoxina, con grado de recomendación IIa (nivel de evidencia C), para el control de la FC en pacientes con insuficiencia cardíaca y disfunción del ventrículo izquierdo ${ }^{(3)}$. El empleo de digoxina se ha evaluado en pacientes con insuficiencia cardíaca y ritmo sinusal, pero no hay ningún ensayo controlado y aleatorizado en el que se haya evaluado la eficacia o la seguridad a largo plazo de la digoxina en pacientes con $\mathrm{FA}^{(2)}$.

En un intento de llenar este vacío, recientemente se han publicado varios análisis observacionales, incluidos análisis post hoc de ensayos clínicos, registros y metanálisis (814). En estos estudios se han obtenido resultados contradictorios, debido posiblemente a las diferencias en la población de pacientes y en los métodos analíticos. La digoxina tiene un margen terapéutico estrecho, y sus niveles se ven influidos de manera sustancial por las interacciones farmacológicas y las comorbilidades ${ }^{(2)}$. Una limitación importante de todos los estudios previos en los que se ha examinado la seguridad de la digoxina en los pacientes con FA es la falta de determinaciones de la concentración sérica de este fármaco, que son necesarias para definir una posible relación entre dosis y respuesta. Un análisis post hoc del ensayo DIG en 1171 pacientes con insuficiencia cardíaca pero sin FA sugirió que la concentración de digoxina en suero estaba directamente relacionada con la mortalidad, y que esta era más baja en los pacientes con niveles de digoxina bajos (de entre 0,5 y 0,8 $\mathrm{ng} / \mathrm{ml}$ ) y aumentaba en los pacientes con niveles $>1,1 \mathrm{ng} / \mathrm{ml}$.

Recientemente fue publicado un análisis post hoc del ensayo ARISTOTLE donde se informa que los pacientes en tratamiento crónico con digoxina en el momento de la aleatorización no presentaron mayor mortalidad aunque sí aquellos con digoxinemia $\geq 1,2$ $\mathrm{ng} / \mathrm{mL}$, aumentándose en un $56 \%$ comparado con pacientes sin digoxina. Cada incremento de la digoxinemia de $0,5 \mathrm{ng} / \mathrm{mL}$ se asoció con un $19 \%$ de aumento de mortalidad tanto en pacientes con insuficiencia cardiaca como sin ella. El inicio tratamiento con digoxina durante el estudio se relacionó con una mayor mortalidad y mayor riesgo de muerte súbita. Estos datos podrían ser suficientes para restringir de una forma muy considerable la prescripción de este fármaco. A pesar de que el estudio se ha planteado siguiendo una metodología impecable no debemos olvidar que se trata de un sub-estudio, no pre-especificado, con grupo control apareado por propensity score y con los posibles sesgos que todo ello implica ${ }^{(4)}$.

La digitalización oral lenta, generalmente preferida para la mayoría de los pacientes, se puede lograr al comenzar una dosis de mantenimiento de 0.125 a $0.25 \mathrm{mg}$ al día. Se logrará un estado estable después de cinco ciclos de vida media del fármaco, que es aproximadamente de 7 a 10 días en el sujeto promedio. La digitalización rápida por vía intravenosa y oral puede usarse para controlar la respuesta ventricular en la fibrilación auricular y el aleteo. Sin embargo, otros medicamentos pueden ser más efectivos y/o tener un inicio de acción más rápido en la respuesta ventricular en estas arritmias; por lo tanto, rara vez se necesita una rápida digitalización, a menos que los medicamentos alternativos estén contraindicados o no hayan sido efectivos. La dosis de carga total con digoxina varía 
de un paciente a otro, pero generalmente está entre 0,75 y $1,5 \mathrm{mg}$ con administración intravenosa y de 1 a $1,5 \mathrm{mg}$ con administración oral ${ }^{(2)}$.

La carga intravenosa para el control de la frecuencia ventricular en la fibrilación auricular y el aleteo, el medio más rápido de digitalización es la vía intravenosa. Se administra una dosis intravenosa inicial de 0,25 a $0,5 \mathrm{mg}$ de digoxina durante varios minutos, seguida de $0,25 \mathrm{mg}$ cada 6 horas para una dosis de carga total de 0,75 a 1,5 mg (10 a $12 \mathrm{mcg} / \mathrm{kg}$ de peso corporal magra)). La digoxina intravenosa comienza a actuar en 15 a 30 minutos con un efecto máximo en 1 a 5 horas. La carga oral mediante la digitalización oral rápida se puede lograr administrando 0,5 mg inicialmente seguido de $0,25 \mathrm{mg}$ cada seis horas para una dosis de carga total de 0,75 a $1,5 \mathrm{mg}^{(2)}$.

Los pacientes que son hipopotasémicos, hipomagnesémicos, hipercalcémicos, hipóxicos o con hipotiroidismo son más sensibles a los efectos de la digoxina. Si estos problemas persisten en el momento de la carga de digoxina, se debe considerar una dosis de carga inicial en el rango inferior ( $p$. Ej., $0,75 \mathrm{mg} o$ menos $)^{(6)}$. La digoxina se distribuye ampliamente en el músculo esquelético, el corazón y otros tejidos magros y tiene un gran volumen de distribución (es decir, 4 a $7 \mathrm{l} / \mathrm{kg}$ ) en sujetos normales, que disminuye en pacientes mayores, tienen baja masa muscular esquelética o insuficiencia renal grave. Las concentraciones séricas de digoxina en relación con la dosis de carga administrada pueden aumentar proporcionalmente entre las personas con masa muscular baja y/o insuficiencia renal, y se debe considerar una dosis de carga reducida. La dosis de carga debería reducirse en aproximadamente un tercio a la mitad en el marco de la insuficiencia renal crónica grave, incluyendo hemodiálisis. La dosis de carga reducida se puede complementar después de seis horas si la respuesta clínica es inadecuada, en ausencia de toxicidad ${ }^{(6)}$.

En general, es razonable seleccionar una dosis de carga inicial en el rango inferior (es decir, 0,75 a $1 \mathrm{mg}$ ) para pacientes con un peso corporal bajo o promedio (por ejemplo, 45 a $70 \mathrm{~kg}$ ) y en la parte superior del rango de dosis (es decir, de 1 a 1,5 mg) para pacientes con un peso corporal medio o superior al promedio (por ejemplo, 71 a $90 \mathrm{~kg}$ ). Los pacientes con un peso corporal inferior a $45 \mathrm{~kg}$ deben recibir el 50 por ciento de la dosis de carga normal. En comparación con sujetos de peso normal, ni el volumen de distribución ni el aclaramiento de digoxina se alteran de manera constante por los cambios en la composición corporal asociados con la obesidad. Por lo tanto, se deben usar dosis de carga normales (sin base de peso). Sin embargo, si se usa una dosis basada en el peso, debe basarse en el peso corporal estimado estimado ${ }^{(6)}$.

Para la mayoría de los pacientes, la dosis de mantenimiento de digoxina será entre 0.125 mg y $0.25 \mathrm{mg}$ al día. La dosis diaria de mantenimiento variará dependiendo de la indicación para la terapia con digoxina, y los pacientes que reciben digoxina para la insuficiencia cardíaca a menudo requieren dosis más bajas que los que toman digoxina para el control de la frecuencia ventricular. Además, la dosis de mantenimiento se ve afectada por la función renal, el peso corporal y la presencia o ausencia de otros medicamentos que se sabe que alteran el metabolismo de la digoxina. Control de frecuencia ventricular para los pacientes que toman digoxina para el control de la frecuencia ventricular en el contexto de las arritmias auriculares, la dosis de mantenimiento típica es entre 0.125 y $0.25 \mathrm{mg}$ al día. A diferencia del uso de digoxina para la insuficiencia cardíaca, no existe un nivel específico de digoxina sérica que sea el objetivo, ya que la medicación debe ajustarse para mantener el control óptimo de la frecuencia ventricular. Sin embargo, se deben evitar niveles séricos de digoxina superiores a $1 \mathrm{ng} / \mathrm{ml}(1,3 \mathrm{nmol} / \mathrm{l})$ para reducir el riesgo de toxicidad por $\operatorname{digoxina}^{(2)}$.

La biodisponibilidad para tabletas de digoxina convencionales está entre 70 y 80 por ciento. A diferencia de otros medicamentos, la biodisponibilidad de las formas de dosis orales de digoxina no parece verse afectada por la insuficiencia cardíaca. Los ajustes de dosis de digoxina son necesarios en pacientes con disfunción renal, pacientes con bajo peso corporal y con el uso concomitante de ciertos medicamentos ${ }^{(6)}$.

Aproximadamente del 70 al 80 por ciento de la digoxina se elimina sin cambios en la orina, lo que lleva a la prolongación de la vida media en pacientes con insuficiencia renal. La insuficiencia renal también disminuye el volumen de distribución extravascular de digoxina, otro efecto que puede elevar los niveles de fármaco en plasma. Como resultado, tanto la dosis de carga inicial como la dosis de mantenimiento deben reducirse en pacientes con enfermedad renal subyacente. En la enfermedad renal terminal, por ejemplo, la dosis de carga debe ser de la mitad a dos tercios de lo normal. La enfermedad hepática tiene poca 
influencia en la digoxina metabolismo o aclaramiento; por lo tanto, no es necesario ajustar la dosis ${ }^{(6)}$.

En la Tabla 4 se observa una serie de interacciones medicamentosas importantes que es necesario tenerlas en cuenta.

Tabla 4: Grupos de interacciones medicamentosas importantes con la digoxina

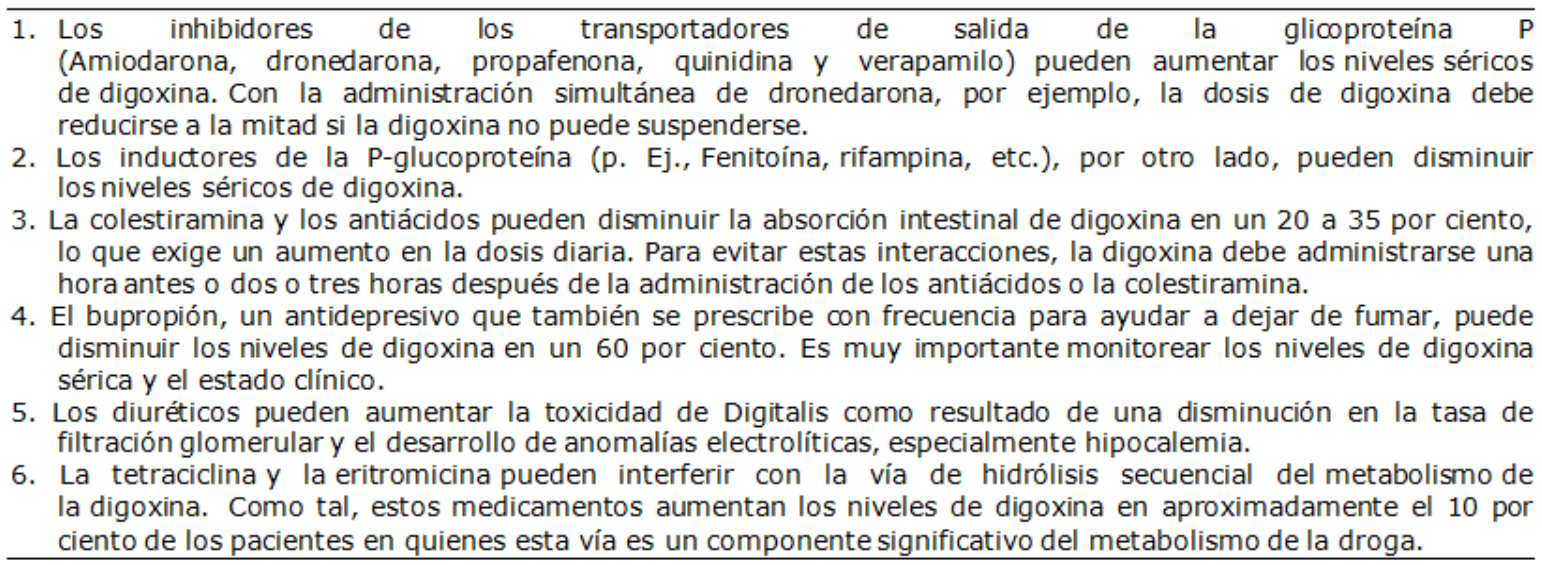

La digoxina atraviesa la placenta y se ha utilizado para indicaciones cardiacas fetales y maternas sin informe de daño fetal o teratogenicidad. Como tal, no hay contraindicaciones para usar digoxina durante el embarazo o durante la lactancia ${ }^{(2)}$. Los efectos inotrópicos de la digoxina generalmente no son beneficiosos en pacientes con amiloidosis. Además, dado que la digoxina se une con avidez a las fibrillas de amiloide, los pacientes con amiloidosis que toman digoxina pueden tener un mayor riesgo de toxicidad por digoxina. Además, como resultado de la unión de digoxina a las fibrillas de amiloide miocárdicas, la concentración de digoxina cardíaca puede ser más alta que la concentración sérica de digoxina, lo que conduce a toxicidad en el establecimiento de niveles "terapéuticos" de digoxina sérica. Sin embargo, en un paciente con fibrilación auricular con una respuesta ventricular rápida, la administración cuidadosa de digoxina suele ser segura y efectiva para reducir la frecuencia ventricular ${ }^{(6)}$.

Dada la ventana terapéutica relativamente estrecha de la digoxina, con un solapamiento sustancial entre los llamados niveles terapéuticos y tóxicos, los pacientes que toman digoxina requieren el control de la concentración sérica de digoxina, y el nivel "óptimo" varía con el entorno clínico. El control del nivel de digoxina sérica es particularmente importante en personas con disfunción renal crónica o función renal que cambia rápidamente, ya que una función renal significativamente disminuida puede conducir a la acumulación de digoxina y sus metabolitos y predisponer a la toxicidad de la digoxina. Además, los pacientes con trastornos electrolíticos, especialmente hipocalemia e hipomagnesemia, que pueden estar relacionados con diuréticos u otros medicamentos, tienen mayor riesgo de arritmias asociadas a digoxina y deben controlar el nivel de digoxina en suero hasta que el nivel sérico de potasio y la concentración de magnesio vuelvan al rango normal ${ }^{(44)}$.

El control de la concentración sérica de digoxina es más importante cuando se usa digoxina en el tratamiento de la insuficiencia cardíaca con disfunción sistólica, mientras que los niveles solo se controlan cuando se usan en pacientes con fibrilación auricular si se sospecha toxicidad. Se deben obtener muestras de sangre al menos 6 horas, pero de manera óptima 12 horas después de la administración de digoxina para asegurar la finalización de la distribución de la sangre a los tejidos. En pacientes con enfermedad renal avanzada o en hemodiálisis, el nivel de digoxina debe controlarse al menos 12 a 24 horas después de la dosis anterior. Las concentraciones séricas de digoxina medidas antes de estos tiempos pueden estar falsamente elevadas ${ }^{(6)}$.

La digoxina generalmente es menos eficaz para controlar la fibrilación auricular (FA) que los beta-bloqueadores o los bloqueadores de los canales de calcio, es menos probable que controle la frecuencia ventricular durante el ejercicio (cuando el tono vagal es bajo y el tono simpático es alto), o sin capacidad para terminar la arritmia, y con frecuencia no disminuye la frecuencia cardíaca con FA recurrente. Por lo tanto, los pacientes con frecuencia 
requieren la adición de un bloqueador beta o bloqueador de los canales de calcio para un control óptimo de la frecuencia ${ }^{(45)}$.

Cuando la digoxina se usa estrictamente para controlar la frecuencia ventricular en la FA, los niveles séricos de digoxina deben controlarse periódicamente, aunque la concentración del fármaco a menudo no se correlaciona con el control de la frecuencia ventricular y se usa más como guía de toxicidad que para la terapia. Los latidos de escape de la unión (detectados por la igualdad de todos los intervalos RR observados más largos en el electrocardiograma) son comunes cuando la digitalización ha disminuido con éxito la frecuencia ventricular. Administrar más digoxina en este contexto aumentará el grado de bloqueo del nodo AV y producirá períodos de ritmo de unión regular. El cambio de escapes de unión única a ritmo de unión periódica generalmente significa el desarrollo de toxicidad por digoxina ${ }^{(47)}$.

\section{Amiodarona}

La amiodarona puede ser útil para el control de la frecuencia cardiaca, pero como último recurso. Los numerosos efectos adversos extracardíacos asociados a la amiodarona hacen que sea más conveniente reservar este fármaco para pacientes cuya frecuencia cardiaca no se puede controlar con tratamiento combinado ${ }^{(48-50)}$. En resumen, hay un equilibrio en el uso de diferentes fármacos para el control de la frecuencia cardiaca en la FA. La elección de un bloqueador beta, diltiazem/verapamilo, digoxina o tratamiento combinado se debe hacer de manera individualizada, teniendo en cuenta las características y las preferencias del paciente. Todos los tratamientos disponibles tienen potenciales efectos adversos. En todos los pacientes se debe iniciar el tratamiento a dosis bajas e ir aumentándolas gradualmente para lograr el alivio de los síntomas. En la práctica, para alcanzar una frecuencia cardiaca $<110$ Ipm suele ser necesario el tratamiento combinado. Se está investigando el beneficio de diferentes estrategias de control de la frecuencia cardiaca en relación con los síntomas, la calidad de vida y otras variables intermedias.

\section{Objetivos de control de la frecuencia cardiaca en la fibrilación auricular}

No está claro cuál es el objetivo óptimo de control de la frecuencia cardiaca de los pacientes con FA. El estudio RACE II incluyo a 614 pacientes, aleatorizados al grupo de control de la frecuencia cardiaca con un objetivo $<80 \mathrm{lpm}$ en reposo y $<110 \mathrm{lpm}$ en ejercicio moderado o al grupo de control menos estricto, con una frecuencia cardiaca $<110 \mathrm{lpm}$. No se observaron diferencias en la variable compuesta de complicaciones clínicas (el 14,9\% en el grupo de control estricto y el $12,9 \%$ en el grupo de control menos estricto), la clase funcional (NYHA) o las hospitalizaciones. En el análisis conjunto de los estudios AFFIRM y RACE (1.091 participantes) se obtuvieron resultados similares, aunque las diferencias en la frecuencia cardiaca fueron más pequeñas y no hubo distribución aleatoria. Hay que destacar que muchos pacientes con un control adecuado de la frecuencia cardíaca (60-100 lpm en reposo) están muy sintomáticos y requieren tratamiento adicional. Sin embargo, un control menos estricto de la frecuencia cardiaca puede ser una estrategia inicial aceptable, independientemente del grado de IC, excepto cuanto los síntomas requieren un control más estricto $^{(7)}$.

\section{Control del ritmo cardiaco en la fibrilación auricular}

Restaurar y mantener el ritmo sinusal es una parte integral del tratamiento de la FA. Los fármacos antiarrítmicos aproximadamente duplican la tasa de pacientes en ritmo sinusal comparados con placebo. La ablación con catéter o el tratamiento combinado suelen ser eficaces en caso de fracaso del tratamiento con antiarrítmicos. Aunque muchos profesionales creen que mantener el ritmo sinusal mejora los resultados en pacientes con FA, todos los estudios que compararon el control de la frecuencia y del ritmo frente al control únicamente de la frecuencia (con anticoagulación) obtuvieron resultados neutros. Por el momento, el tratamiento para el control del ritmo está indicado para mejorar los síntomas de los pacientes con FA que siguen sintomáticos a pesar de recibir un tratamiento adecuado para el control de la frecuencia $\operatorname{cardiaca}^{(44-47)}$.

Los fármacos antiarrítmicos pueden restablecer el ritmo sinusal en pacientes con FA. La dofetilida, un fármaco con capacidad para revertir la FA de reciente aparición ${ }^{(66-68)}$. La cardioversión farmacológica puede restaurar el ritmo sinusal en, aproximadamente, el 50\% de los pacientes con FA de reciente aparición. A corto plazo, la cardioversión eléctrica restaura el ritmo sinusal más rápida y eficazmente que la cardioversión farmacológica y se asocia con hospitalizaciones más cortas. En cambio, la cardioversión farmacológica no requiere sedación ni ayuno ${ }^{(7)}$. 
La flecainida y la propafenona son eficaces para la cardioversión farmacológica, pero su uso está restringido a pacientes sin cardiopatía estructural ${ }^{(57-59)}$. La ibutilida es una alternativa, si está disponible, pero implica un riesgo de torsades de pointes. El vernakalant se puede administrar a pacientes con IC leve (NYHA I-II), incluidos los pacientes con cardiopatía isquémica, siempre que no presenten hipotensión o estenosis aortica grave. La amiodarona se puede emplear en pacientes con IC o cardiopatía isquémica. Sin embargo, la mayoría de los estudios sobre la cardioversión de la FA excluyeron a los pacientes con IC grave. La amiodarona también ralentiza la frecuencia cardiaca en 10-12 lpm después de 8$12 \mathrm{~h}$ cuando se administra por vía intravenosa. Tanto la amiodarona como la flecainida parecen ser más efectivas que el sotalol para la restauración del ritmo sinusal ${ }^{(46)}$.

Para pacientes con episodios poco frecuentes de FA paroxística seleccionados, se puede indicar la autoadministración (estrategia de pastilla en el bolsillo, "pill in the pocket") de un bolo oral de flecainida $(200-300 \mathrm{mg})$ o propafenona $(450-600 \mathrm{mg})$ para restaurar el ritmo sinusal, una vez que se haya establecido la seguridad de esta estrategia en el hospital. Esta opción es marginalmente menos efectiva que la cardioversión en el hospital, pero es práctica y proporciona control y tranquilidad a algunos pacientes seleccionados ${ }^{(7)}$. La cardioversión eléctrica directa sincronizada revierte rápida y eficazmente la FA a ritmo sinusal y es el método de elección para pacientes con grave deterioro hemodinámico y FA de nueva aparición. La cardioversión eléctrica se puede realizar con seguridad en pacientes sedados tratados con midazolam intravenoso o propofol. Durante el procedimiento es importante la monitorización continua de la presión arterial y la oximetría. En algunas ocasiones se producen quemaduras cutáneas. Se debe disponer de atropina o isoproterenol intravenosos, o marcapasos transcutáneo temporal, para mitigar la bradicardia después de la cardioversión. Los desfibriladores bifásicos son más efectivos que los monofásicos y, hoy en día, son los más empleados. La posición anteroposterior del electrodo genera un campo de choque en la aurícula derecha más fuerte que con la posición anterolateral, y es más efectiva para la restauración del ritmo sinusal ${ }^{(7)}$.

El pretratamiento con amiodarona (varias semanas), sotalol, ibutilida o vernakalant puede mejorar la eficacia de la cardioversión eléctrica; se han observado efectos similares con flecainida y propafenona. Los bloqueadores beta, verapamilo, diltiazem y digoxina no son fármacos fiables para terminar la FA ni facilitan la cardioversión eléctrica. Cuando se planifica el tratamiento antiarrítmico para mantener el ritmo sinusal tras la cardioversión, parece prudente empezar el tratamiento 1-3 días antes de la cardioversión (con amiodarona, varias semanas) para facilitar la cardioversión farmacológica y alcanzar concentraciones del fármaco efectivas.

La cardioversión conlleva un riesgo inherente de ACV en pacientes no anticoagulados, lo cual se puede reducir sustancialmente con la administración de anticoagulantes orales (ACO). Es importante iniciar inmediatamente la anticoagulación de pacientes programados para cardioversión. Los pacientes que se han mantenido en FA durante más de 48 h deben comenzar la anticoagulación al menos 3 semanas antes de la cardioversión y después continuar el tratamiento durante 4 semanas (los que no requieran anticoagulación crónica). Los ACO se deben mantener indefinidamente a los pacientes con riesgo de ACV. Esta práctica no se ha evaluado en estudios con grupo de control, pero según los datos observacionales de una gran base de datos finlandesa su uso es seguro. Cuando se desea realizar una cardioversión precoz, la ETE puede excluir la mayoría de los trombos en la aurícula izquierda y permitir la cardioversión inmediata. Los estudios que se desarrollan actualmente proporcionaran información sobre la seguridad y la eficacia de los nuevos tratamientos con los nuevos ACO en pacientes programados para cardioversión ${ }^{(7)}$.

\section{Tratamiento antiarrítmico a largo plazo}

El objetivo del tratamiento con fármacos antiarrítmicos es mejorar los síntomas relacionados con la FA. Por ello, la decisión de iniciar tratamiento antiarrítmico a largo plazo debe buscar un equilibrio entre la carga sintomática, la posibilidad de reacciones farmacológicas adversas y las preferencias del paciente. Los principios del tratamiento antiarrítmico propuestos en la guía de la ESC sobre FA (2010) y que se observan en la Tabla 5 siguen siendo relevantes y deben ser observados. 
Tabla 5: Principios del tratamiento antiarrítmico propuestos en la guía de la ESC sobre FA

1. El tratamiento está dirigido a reducir los síntomas relacionados con la FA.

2. La eficacia de los fármacos antiarrítmicos para mantener el ritmo sinusal es moderada.

3. El tratamiento antiarrítmico farmacológico que tiene éxito clínico puede reducir pero no eliminar la recurrencia de la FA.

4. Si un fármaco antiarrítmico fracasa, se puede obtener una respuesta clínica aceptable con otro fármaco.

5. Las proarritmias inducidas por fármacos o los efectos secundarios cardiacos son frecuentes.

6. Las consideraciones sobre seguridad, más que la eficacia, deben ser la principal guía en la elección del fármaco antiarrítmico.

7. El tratamiento con fármacos antiarrítmicos prácticamente duplica el mantenimiento del ritmo sinusal comparado con la ausencia de tratamiento.

No se ha apreciado una diferencia respecto a la mortalidad o las complicaciones CV, pero el tratamiento antiarrítmico puede incrementar ligeramente el riesgo de hospitalización (normalmente por FA). Para reducir el riesgo de efectos secundarios, es conveniente que la duración del tratamiento sea corta.

Por ejemplo, el tratamiento con flecainida durante 4 semanas tras la cardioversión de la FA tuvo buena tolerancia y previno la mayoría de las recurrencias de FA (80\%), comparado con el tratamiento a largo plazo ${ }^{(57-59)}$. El tratamiento antiarrítmico a corto plazo también se emplea para evitar la recurrencia de la FA tras la ablación con catéter $y$, además, puede ser una opción razonable para los pacientes con mayor riesgo de sufrir efectos secundarios o con bajo riesgo percibido de FA recurrente. Además del tratamiento antiarrítmico y la ablación con catéter, el tratamiento de enfermedades CV concomitantes puede reducir la carga sintomática de la FA y facilitar el mantenimiento del ritmo sinusal. Esto incluye la pérdida de peso, el control de la presión arterial, el tratamiento de la IC, el aumento de la capacidad cardiorrespiratoria y otras medidas ${ }^{(7)}$.

\section{Selección de fármacos antiarrítmicos para el tratamiento a largo plazo}

Normalmente, la seguridad del tratamiento antiarrítmico determina la elección inicial de que fármacos emplear. Los siguientes fármacos están disponibles para prevenir la FA.

\section{Amiodarona}

La amiodarona es un eficaz bloqueador multicanal que reduce la frecuencia ventricular y es segura en pacientes con IC. Se puede producir proarritmia secundaria a torsades de pointes, y durante el tratamiento es preciso monitorizar el intervalo QT y las ondas T y ondas $U$. La amiodarona causa frecuentemente efectos secundarios extracardíacos, especialmente cuando se emplea a largo plazo, por lo que se lo considera un fármaco de segunda línea para los pacientes candidatos a otros fármacos antiarrítmicos. La amiodarona parece ser menos adecuada para el tratamiento de episodios a corto plazo (excepto tras la ablación con catéter), probablemente debido a su larga vida media biológica ${ }^{(48-50)}$.

\section{Dronedarona}

La dronedarona mantiene el ritmo sinusal, reduce la frecuencia ventricular y previene las hospitalizaciones de causa CV (fundamentalmente por FA) y la muerte CV de pacientes con FA paroxística o persistente que además tienen, como mínimo, una comorbilidad CV relevante. La dronedarona aumenta la mortalidad de los pacientes con IC recientemente descompensada (con o sin FA) y los pacientes con FA permanente en los que no se restablece el ritmo sinusal. La dronedarona aumenta moderadamente la creatinina sérica, que refleja más una reducción de la excreción de creatinina que un deterioro de la función renal $^{(51-53)}$.

\section{Flecainida y propafenona}

La flecainida y la propafenona son efectivas para la prevención de la $F A^{(54-56)}$. Solo deben emplearse en pacientes sin cardiopatía isquémica significativa ni IC para evitar el riesgo de arritmias ventriculares potencialmente mortales. La frecuencia ventricular elevada secundaria a la conversión de la FA en flutter auricular con conducción $1: 1$ que se produce por la administración de flecainida o propafenona se puede prevenir con la administración previa de un bloqueador beta, verapamilo o diltiazem ${ }^{(57-59)}$.

\section{Quinidina y disopiramida}

La quinidina y la disopiramida se han asociado con un aumento de la mortalidad por todas las causas al año de seguimiento, probablemente debido a arritmias ventriculares (torsades de pointes). Aunque este efecto proarrítmico es más común a dosis altas, estos fármacos se 
emplean con menos frecuencia para el control del ritmo cardiaco en la FA. La disopiramida puede ser útil en la FA mediada por un efecto vagal (FA en atletas o durante el sueño), y se ha demostrado que reduce el gradiente del flujo de salida del VI y mejora los síntomas de los pacientes con miocardiopatía hipertrófica ${ }^{(60-62)}$.

\section{Sotalol}

El sotalol conlleva un riesgo importante de torsades de pointes. Su d-enantiómero se ha asociado con un aumento de la mortalidad, comparado con placebo, en pacientes con disfunción del VI tras infarto de miocardio, probablemente debido a arritmias ventriculares (OR $=2,47$; IC95\%, 1,2-5,05; NNP = 166; IC95\%,61-1.159). Por otra parte, el d,l-sotalol se ha empleado en pacientes con FA sin que se observaran problemas de seguridad en 2 estudios con grupo de control ${ }^{(63-65)}$.

\section{Dofetilida}

La dofetilida es otro bloqueador de los canales de potasio que está disponible fundamentalmente en países no europeos. La dofetilida restablece y mantiene el ritmo sinusal de pacientes con IC $y$, en algunas ocasiones, también en pacientes que son refractarios a otros antiarrítmicos ${ }^{(66-68)}$. En general, parece prudente limitar el uso de quinidina, disopiramida, dofetilida y sotalol a situaciones específicas. Además, la combinación de antiarrítmicos que prolongan el intervalo QT no se debe emplear para el control del ritmo cardiaco en la FA.

\section{CONCLUSIÓN}

La seguridad del tratamiento antiarrítmico determina la elección inicial de que fármacos emplear. El objetivo del tratamiento con fármacos antiarrítmicos es mejorar los síntomas relacionados con la FA. Por ello, la decisión de iniciar tratamiento antiarrítmico a largo plazo debe buscar un equilibrio entre la carga sintomática, la posibilidad de reacciones farmacológicas adversas y las preferencias del paciente. El control de la frecuencia es una parte integral del tratamiento de los pacientes con FA y, normalmente, es suficiente para mejorar los síntomas relacionados con ella. El control de la frecuencia cardiaca a corto y largo plazo se puede lograr con tratamiento farmacológico, que incluya bloqueadores beta, digoxina, bloqueadores de los canales del calcio o tratamiento combinado. Si bien otros fármacos antiarrítmicos de clase III también tienen un efecto limitador de la frecuencia, solo deben emplearse en pacientes que necesitan tratamiento para el control del ritmo cardiaco.

Restaurar y mantener el ritmo sinusal es una parte integral del tratamiento de la FA. Los fármacos antiarrítmicos de clase III duplican la tasa de pacientes en ritmo sinusal. El tratamiento para el control del ritmo está indicado para mejorar los síntomas de los pacientes con FA que siguen sintomáticos pese a recibir un tratamiento adecuado para el control de la frecuencia cardiaca. La combinación de antiarrítmicos que prolongan el intervalo QT no se debe emplear para el control del ritmo cardiaco en la FA.

\section{REFERENCIAS BIBLIOGRÁFICAS}

1. Centurión OA, Aquino NJ, García LB, Torales JM. Idarucizumab humanized monoclonal antibody fragment, for reversal of Dabigatran therapy for atrial fibrillation. Blood Heart Circ. 2017;1:DOI:10.15761/BHC:1000105.

2. Lopes R, Rordorf R, De Ferrari G, Leonardi S, Thomas L, Wojdyla $D$, et al. Digoxina $y$ mortalidad en la fibrilación auricular. J Am Coll Cardiol 2018;71:1063-74.

3. Nguyen TN, Hilmer SN, Cumming RG. Review of epidemiology and management of atrial fibrillation in developing countries. Int J Cardiol. 2013;167:2412-20.

4. Oldgren J, Healey JS, Ezekowitz M, Commerford P, Avezum A, Pais P, et al. RELY Atrial Fibrillation Registry Investigators. Variations in cause and management of atrial fibrillation in a prospective registry of 15,400 emergency department patients in
46 countries: the RE-LY Atrial Fibrillation Registry. Circulation. 2014;129:1568-76.

5. Chiang $C E$, Naditch-Brule $L$, Murin J, Goethals M, Inoue $H$, O'Neill $J$, et al. Distribution and risk profile of paroxysmal, persistent, and permanent atrial fibrillation in routine clinical practice: insight from the real-life global survey evaluating patients with atrial fibrillation international registry. Circ Arrhythm Electrophysiol. 2012;5:63239.

6. Guerra $F$, Brambatti $M$, Nieuwlaat $R$, Marcucci M, Dudink E, Crijns HJGM, et al. Symptomatic atrial fibrillation and risk of cardiovascular events: data from the Euro Heart Survey. Europace. 2017;19(12):192229.

7. Kirchhof $P$ et al. Grupo de Trabajo de la Sociedad Europea de Cardiología (ESC) para el diagnóstico y tratamiento de la fibrilación 
auricular. Rev Esp Cardiol 2017; 70(1):50.e1-e84.

8. Ziff OJ, Lane DA, Samra M, Griffith $M$, Kirchhof P, Lip GY, et al. Safety and efficacy of digoxin: systematic review and meta-analysis of observational and controlled trial data. BMJ. 2015;351:h4451.

9. Khand AU, Rankin AC, Martin W, Taylor J, Gemmell I, Cleland JG. Carvedilol alone or in combination with digoxin for the management of atrial fibrillation in patients with heart failure? J Am Coll Cardiol. 2003; 42:1944-51.

10. Van Gelder IC, Groenveld HF, Crijns HJ, Tuininga YS, Tijssen JG, Alings AM, et al. Lenient versus strict rate control in patients with atrial fibrillation. $\mathrm{N}$ Engl J Med. 2010;362:1363-73.

11. Groenveld HF, Crijns HJ, Van den Berg MP, Van Sonderen E, Alings AM, Tijssen JG, et al. The effect of rate control on quality of life in patients with permanent atrial fibrillation: data from the RACE II (Rate Control Efficacy in Permanent Atrial Fibrillation II) study. J Am Coll Cardiol. 2011;58:1795-803.

12. Zi OJ, Kotecha D. Digoxin: the good and the bad. Trends Cardiovasc Med 2016;26: 585-95.

13. Carlsson J, Miketic S, Windeler J, Cuneo A, Haun S, Micus $S$, et al. Randomized trial of rate-control versus rhythm-control in persistent atrial fibrillation: the Strategies of Treatment of Atrial Fibrillation (STAF) study. J Am Coll Cardiol. 21 de mayo de 2003;41(10):1690-6.

14. Centurión OA, Shimizu A. Rate Control Strategy Elevated To Primary Treatment For Atrial Fibrillation: Has The Last Word Already Been Spoken? J Atr Fibrillation $2015 ; 7(4): 50-6$

15. Centurión OA. Atrial fibrillation in the Wolff-Parkinson-White syndrome. J Atr Fibrillation 2011;4(1): 287.

16. Centurión OA, Torales JM, García LB. Sudden Cardiac Death Associated to Auriculoventricular Accessory Pathways: Sleeping with the Enemy. EC Cardiology 2018; 5(9):612-15.

17. Centurión $O A$, Shimizu A, Isomoto $S$, Konoe A. Mechanisms for the genesis of paroxysmal atrial fibrillation in the WolffParkinson-White syndrome: Intrinsic atrial muscle vulnerability vs. electrophysiological properties of the accessory pathway. Europace $2008 ; 10(3)$ : 294-302.

18. Wellens HJJ and Durrer D. Wolff-ParkinsonWhite syndrome and atrial fibrillation. Relation between refractory period of the accessory pathway and ventricular rate during atrial fibrillation. Am J Cardiol 1974;34(7):772-82.

19. Waspe LE, Brodman R, Kim SG, Fisher JD. Susceptibility to atrial fibrillation and ventricular tachyarrhythmia in the WolffParkinson-White syndrome: Role of the accessory pathway. Am Heart J 1986;112(6): 1141-52.

20. Centurión OA, Scavenius KE, García LB, Miño L, Torales J, Sequeira O. Atrioventricular Nodal Catheter Ablation in Atrial Fibrillation Complicating Congestive Heart Failure. J Atr Fibrillation. 2018 Jun 30;11(1):1813. doi: 10.4022/jafib.1813.

21. Palmisano $\mathrm{P}$, Aspromonte $\mathrm{V}$, Ammendola $\mathrm{E}$, Dell'era G, Ziacchi M, Guerra F, et al. Effect of fixed-rate vs. rate-RESPONSIve pacing on exercise capacity in patients with permanent, refractory atrial fibrillation and left ventricular dysfunction treated with atrioventricular junction aBLation and bivEntricular pacing (RESPONSIBLE): a prospective, multicentre, randomized, single-blind study. Europace. 2017;19 (3):414-20.

22. Ringwala $S$, Knight BP, Verma N. Permanent His bundle pacing at the time of atrioventricular node ablation: A 3dimensional mapping approach. HeartRhythm Case Rep. 2017;3 (6):323325.

23. Occhetta $E$, Bortnik $M$, Magnani $A$, Francalacci G, Piccinino C, Plebani $L$, Marino $P$. Prevention of ventricular desynchronization by permanent paraHisian pacing after atrioventricular node ablation in chronic atrial fibrillation: a crossover, blinded, randomized study versus apical right ventricular pacing. J. Am. Coll. Cardiol. 2006;47(10):1938-45.

24. Huang W, Su L, Wu S, Xu L, Xiao F, Zhou $X$, et al. Benefits of Permanent His Bundle Pacing Combined With Atrioventricular Node Ablation in Atrial Fibrillation Patients With Heart Failure With Both Preserved and Reduced Left Ventricular Ejection Fraction. J Am Heart Assoc. 2017;6 (4). pii: e005309. doi: 10.1161/JAHA.116.005309.

25. Björkenheim $A$, Brandes $A$, Andersson $T$, Magnuson A, Edvardsson N, Wandt $B$, et al. Predictors of hospitalization for heart failure and of all-cause mortality after atrioventricular nodal ablation and right ventricular pacing for atrial fibrillation. Europace. 2014;16 (12):1772-78.

26. Hohendanner $F$, Heinzel FR, Blaschke F, Pieske BM, Haverkamp W, Boldt HL, et al.Pathophysiological and therapeutic implications in patients with atrial fibrillation and heart failure. Heart Fail Rev. $2018 ; 23$ (1):27-36.

27. Carson PE, Johnson GR, Dunkman WB, Fletcher RD, Farrell L, Cohn JN. The influence of atrial fibrillation on prognosis in mild to moderate heart failure. The $\mathrm{V}$ HeFT Studies. The V-HeFT VA Cooperative Studies Group. Circulation. 1993;87 (6 Suppl):VI102-110.

28. Modin D, Sengeløv M, Jørgensen PG, Bruun $N E$, Olsen FJ, Dons $M$, et al. Global 
longitudinal strain corrected by RR interval is a superior predictor of all-cause mortality in patients with systolic heart failure and atrial fibrillation. ESC Heart Fail. 2018;5 (2):311-18.

29. Birnie D, Hudnall H, Lemke B, Aonuma K, Lee $\mathrm{KL}$, Gasparini $M$, et al. Continuous optimization of cardiac resynchronization therapy reduces atrial fibrillation in heart failure patients: Results of the Adaptive Cardiac Resynchronization Therapy Trial. Heart Rhythm. 2017;14 (12):1820-25.

30. Centurión OA. Atrial Fibrillation Complicating Congestive Heart Failure: Electrophysiological Aspects And Its Deleterious Effect On Cardiac Resynchronization Therapy. J Atr Fibrillation. 2009;2(1):143. doi: 10.4022/jafib.143.

31. Krijthe BP, Kunst A, Benjamin EJ, Lip GY, Franco $\mathrm{OH}$, Hofman $A$, et al. Projections on the number of individuals with atrial fibrillation in the European Union, from 2000 to 2060. Eur Heart J. 2013;34:274651.

32. Zoni-Berisso M, Lercari F, Carazza T, Domenicucci $S$. Epidemiology of atrial fibrillation: European perspective. Clin Epidemiol. 2014;6:213-20.

33. Bjorck S, Palaszewski B, Friberg L, Bergfeldt L. Atrial fibrillation, stroke risk, and warfarin therapy revisited: a population-based study. Stroke. 2013;44:3103-08.

34. Haim M, Hoshen $M$, Reges O, Rabi $Y$, Balicer R, Leibowitz M. Prospective national study of the prevalence, incidence, management and outcome of a large contemporary cohort of patients with incident non-valvular atrial fibrillation. J Am Heart Assoc. 2015;4:e001486.

35. McManus DD, Rienstra M, Benjamin EJ. An update on the prognosis of patients with atrial fibrillation. Circulation. 2012;126:143-46.

36. Pant R, Patel M, Garcia-Sayan E, Wassouf $M, D^{\prime}$ Silva $O$, Kehoe RF, et al. Impact of Btype natriuretic peptide level on the risk of left atrial appendage thrombus in patients with nonvalvular atrial fibrillation: a prospective study. Cardiovascular Ultrasound 2016; 14: 4 DOI 10.1186/s12947-016-0047-6.

37. Ball J, Carrington MJ, McMurray JJ, Stewart S. Atrial fibrillation: profile and burden of an evolving epidemic in the 21 st century. Int J Cardiol. 2013;167:1807-24.

38. Chugh SS, Havmoeller R, Narayanan K, Singh D, Rienstra M, Benjamin EJ, et al. Worldwide epidemiology of atrial fibrillation: a Global Burden of Disease 2010 Study. Circulation. 2014;129:837-47.

39. Colilla S, Crow A, Petkun W, Singer DE, Simon $\mathrm{T}$, Liu $\mathrm{X}$. Estimates of current and future incidence and prevalence of atrial fibrillation in the U.S. adult population. Am J Cardiol. 2013;112:1142-47.

40. Heeringa J, van der Kuip DA, Hofman A, Kors JA, van Herpen G, Stricker BH, et al. Prevalence, incidence and lifetime risk of atrial fibrillation: the Rotterdam study. Eur Heart J. 2006;27:949-53.

41. Lloyd-Jones DM, Wang TJ, Leip EP, Larson MG, Levy D, Vasan RS, et al. Lifetime risk for development of atrial fibrillation: the Framingham Heart Study. Circulation. 2004; 110:1042-46.

42. Go AS, Hylek EM, Phillips KA, Chang Y, Henault LE, Selby JV, et al.Prevalence of diagnosed atrial fibrillation in adults: national implications for rhythm management and stroke prevention: the AnTicoagulation and Risk Factors in Atrial Fibrillation (ATRIA) Study. JAMA. 2001;285:2370-75.

43. Wyse DG, Waldo AL, DiMarco JP, Domanski $M J$, Rosenberg $Y$, Schron EB, et al. A comparison of rate control and rhythm control in patients with atrial fibrillation. The atrial fibrillation follow-up investigation of rhythm management (AFFIRM) investigators. N Engl J Med 2002;347:1825-33.

44. Van Gelder IC, Hagens VE, Bosker HA, Kingma JH, Kamp O, Kingma T, et al. A comparison of rate control and rhythm control in patients with recurrent persistent atrial fibrillation. $\mathrm{N}$ Engl J Med 2002;347:1834.

45. Opolski G, Torbicki A, Kosior D, Szulc M, Zawadzka M, Pierścińska M,et al. Rhythm control versus rate control in patients with persistent atrial fibrillation. Results of the HOT CAFE Polish Study. Kardiol Pol 2003;59(7):1-16.

46. Honloser SH, Kuck KH, Lilienthal J. Rhythm or rate control in atrial fibrillationPharmacological intervention in atrial fibrillation (PIAF). A randomized trial. Lancet 2000;356:1789-94.

47. Habibollahi $\mathrm{P}$, Jam SH, Vahdati SS, Baghi $\mathrm{HM}$, Amiri $\mathrm{H}$. Amiodarone in atrial fibrillation: post coronary artery bypass graft. World J Emerg Med 2016;7(4):25054.

48. Naccarelli GV, Wolbrette DL, Dell'Orfano JT, Patel HM, Luck JC. Amiodarone: What Have We Learned from Clinical Trials? Clin. Cardiol. 2000;23:73-82.

49. Zipes DP, Prystowsky EN, Heger JJ. Amiodarone: Electrophysiologic Actions, Pharmacokinetics and Clinical Effects. J Am Coll Cardiol 1984;3(4):1059-71.

50. Wolbrette D, Gonzalez M, Samii S, Banchs J, Penny-Peterson E, Naccarelli G. Dronedarone for the treatment of atrial fibrillation and atrial flutter: approval and efficacy. Vasc Health Risk Manag 2010;6:517-23. 
51. Reiffel JA. Dronedarone: Where Does it Fit in the AF Therapeutic Armamentarium?

52. J Atr Fibrillation 2013;5(6):157-63.

53. Naccarelli GV, Kowey PR. The Role of Dronedarone in the Treatment of Atrial Fibrillation/Flutter in the Aftermath of PALLAS. Curr Cardiol Rev 2014;10:3038.

54. Andrikopoulos GK, Pastromas S, Tzeis S. Flecainide: Current status and perspectives in arrhythmia management. World J Cardiol 2015;7(2):76-85.

55. Aliot E, Capucci A, Crijns HJ, Goette A, Tamargo J. Twenty-five years in the making: flecainide is safe and effective for the management of atrial fibrillation. Europace 2011;13:161-73.

56. Juan Tamargo J, Le Heuzey JY, Mabo P. Narrow therapeutic index drugs: a clinical pharmacological consideration to flecainide. Eur J Clin Pharmacol 2015;71:549-67.

57. Camm AJ, Savelieva I. Some patients with paroxysmal atrial fibrillation should carry flecainide or propafenone to self treat. BMJ 2007:334:637. doi: 10.1136/bmj.39143.720602.BE

58. Khairy $P$, Nattel $S$. New insights into the mechanisms and management of atrial fibrillation. Can Med Assoc J 2002;167(9):1012-20.

59. Lip GYH, Apostolakis S. Atrial fibrillation (acute onset). Clinical Evidence 2014;11:210-253.

60. O'Neal Humpries J. Unexpected Instant Death Following Successful Coronary Artery Bypass Graft Surgery (and Other Clinical Settings): Atrial Fibrillation, Quinidine, Procainamide, et cetera, and Instant Death. Clin. Cardiol. 1998;21:711-18.
61. Burashnikov A, Antzelevitch C. New Pharmacological Strategies for the Treatment of Atrial Fibrillation. Ann Noninvasive Electrocardiol. 2009;14(3):290-300.

62. Mackstaller LL, Alpert J. Atrial Fibrillation: A Review of Mechanism, Etiology, and Therapy. Clin. Cardiol. 1997;20:640-50.

63. Batul SA, Gopinathannair R. Intravenous Sotalol - Reintroducing a Forgotten Agent to The Electrophysiology Therapeutic Arsenal. J Atr Fibrillation 2017;9(5):1-5.

64. Chaki AL, Caines AE, Miller AB. Sotalol as Adjunctive Therapy to Implantable Cardioverter-Defibrillators in Heart Failure Patients. Congest Heart Fail. 2009;15:144-47.

65. Marcus FI. Risks of Initiating Therapy With Sotalol for Treatment of Atrial Fibrillation. J Am Coll Cardiol 1998;32(1):177-80.

66. Pedersen OD, Brendorp B, Køber L, Torp-Pedersen C. Prevalence, Prognostic Significance, and Treatment of Atrial Fibrillation in Congestive Heart Failure With Particular Reference to the DIAMOND-CHF Study. CHF. 2003;9:333-40.

67. Jaiswal A, Goldbarg S. Dofetilide induced torsade de pointes: Mechanism, risk factors and management strategies. Indian Heart J 2014;66:64048.

68. Ellison KE, Stevenson WG, Sweeney MO, Epstein LM, Maisel WH. Management of Arrhythmias in Heart Failure. CHF. 2003;9:91-9. 